\title{
On phase transitions of the Potts model with three competing interactions on Cayley tree
}

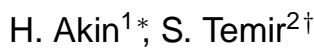 \\ ${ }^{1}$ Department of Mathematics, Faculty of Education, Zirve University, 27260 Gaziantep, Turkey \\ 2 Department of Mathematics, Arts and Science Faculty Harran University, 63200 Sanliurfa, Turkey
}

Received November 15, 2010, in final form March 22, 2011

\begin{abstract}
In the present paper we study a phase transition problem for the Potts model with three competing interactions, the nearest neighbors, the second neighbors and triples of neighbors and non-zero external field on Cayley tree of order two. We prove that for some parameter values of the model there is phase transition. We reduce the problem of describing by limiting Gibbs measures to the problem of solving a system of nonlinear functional equations. We extend the results obtained by Ganikhodjaev and Rozikov [Math. Phys. Anal. Geom., 2009, 12, No. 2, 141-156] on phase transition for the Ising model to the Potts model setting.
\end{abstract}

Key words: phase transition, Potts model, competing interactions, Gibbs measure

PACS: $05.50 .+q, 64.60 .-i, 64.60 . D e, 75.10 . H k$

\section{Introduction}

It is well known that lattice spin system is a large class of systems considered in statistical mechanic. It is also well known that the structure of the lattice plays an important role in examining the spin systems. The Potts model, which was introduced by Potts (in 1952) as a generalization of the Ising model, has drawn an increased attention to its theoretical and experimental aspects in recent years [1]. The idea originated from the representation of the Ising model as interacting spins which can be either parallel or antiparallel.

We consider a semi-infinite Cayley tree $\mathrm{J}^{k}$ of order $k \geqslant 2$, i.e., a graph without cycles with $(k+1)$ edges issuing from each vertex except $x^{0}$ and with $k$ edges issuing from the vertex $x^{0}$, which is called the tree root. We generalize the problem in [2] for the Potts model with three competing interactions and non-zero external field. This problem has been investigated for the Potts model with only two competing interactions [2, 3].

There are many approaches to the derivation of an equation or a system of equations describing the limiting Gibbs measure for the lattice models on Cayley tree (see [1, 4 [9] for details). One of these approaches is based on recursive equations for partition functions [1]. Another approach is Markov random fields (see [10, 11]). Naturally, both approaches lead to the same equation [10].

In this paper we employ the recursive equations for partition functions. We study the problem of phase transition for the Potts model with three competing interactions, the nearest neighbors, the second neighbors and triples of neighbors and non-zero external field on Cayley tree of order two. In [12], it has been proved that there is a phase transition for some parameter values of the Ising model. We generalize this problem for the Potts model. In order to construct the Hamiltonian equation, we consider the generalized Kroneker's $\delta$ function which well agrees with theory of quadratic stochastic operators.

The paper is organized as follows. In section 2 we present basic definitions and notations. In section 3 we present the recursive equations for partition functions. Section 4 contains the solutions of the system of nonlinear equations. These solutions describe the presence or the absence of a phase transition. Finally, the conclusions are presented in section 5 .

\footnotetext{
*E-mail: hasanakin69@gmail.com

${ }^{\dagger}$ E-mail: temirseyit@harran.edu.tr
} 


\section{Preliminaries}

Cayley tree. The Cayley tree $\Gamma^{k}$ (see [5]) of order $k \geqslant 1$ is an infinite tree, i.e., a graph without cycles, from each vertex of which exactly $k+1$ edges issue. Let $\Gamma^{k}=(V, L, i)$, where $V$ is the set of vertices of $\Gamma^{k}, L$ is the set of edges of $\Gamma^{k}$ and $i$ is the incidence function associating each edge $\ell \in L$ with its end points $x, y \in V$. If $i(\ell)=\{x, y\}$, then $x$ and $y$ are called the nearest neighboring vertices and we write $\ell=\langle x, y\rangle$. For $x, y \in V$, the distance $d(x, y)$ on Cayley tree is defined by the formula

$$
\begin{aligned}
d(x, y)= & \min \left\{d \mid x=x_{0}, x_{1}, x_{2}, \ldots, x_{d-1}, x_{d}=y \in V\right. \text { such that the pairs } \\
& \left.\left\langle x_{0}, x_{1}\right\rangle, \ldots,\left\langle x_{d-1}, x_{d}\right\rangle \text { are neighboring vertices }\right\}
\end{aligned}
$$

For the fixed $x^{0} \in V$ we have

$$
\begin{aligned}
W_{n} & =\left\{x \in V: d\left(x^{0}, x\right)=n\right\} \\
V_{n} & =\left\{x \in V: d\left(x^{0}, x\right) \leqslant n\right\} \\
L_{n} & =\left\{\ell=\langle x, y\rangle \in L: x, y \in V_{n}\right\} .
\end{aligned}
$$

A collection of the pairs $\left\langle x_{0}, x_{1}\right\rangle, \ldots,\left\langle x_{d-1}, y\right\rangle$ is called a path from $x$ to $y$. We write $x<y$ if the path from $x^{0}$ to $y$ goes through $x$. We call the vertex $y$ a direct successor of $x$, if $y>x$ and $x, y$ are the nearest neighbors. The set of direct successors of $x$ is denoted by $S(x)$, i.e.,

$$
S(x)=\left\{y \in W_{n+1}: d(x, y)=1\right\}, \quad x \in W_{n} .
$$

It is clear that for any vertex $x \neq x^{0}, x$ has $k$ direct successors and $x^{0}$ has $k+1$. The vertices $x$ and $y$ are called the second neighbor which is denoted by $\rangle x, y\langle$, if there exists a vertex $z \in V$ such that $x, z$ and $y, z$ are the nearest neighbors.

The second neighbors are called one-level neighbors, if vertices $x$ and $y$ belong to $W_{n}$ for some $\mathrm{n}$, that is, if they are located on the same level. We will consider only one-level second neighbors. Three vertices $x, y$ and $z$ are called a triple of neighbors, which is denoted by $\langle x, y, z\rangle$, if $\langle x, y\rangle$ and $\langle y, z\rangle$ are nearest neighbors and $x \neq z$.

For neighbors triple $\langle x, y, z\rangle$ we consider the following Kroneker's symbol

$$
\delta_{\sigma(x) \sigma(y) \sigma(z)}=\left\{\begin{array}{cc}
1, & \text { if } \sigma(x)=\sigma(y)=\sigma(z), \\
1 / 2, & \text { if } \sigma(x)=\sigma(y) \neq \sigma(z) \\
0, & \text { otherwise, }
\end{array} \text { or } \sigma(x) \neq \sigma(y)=\sigma(z),\right.
$$

where $x, z \in W_{n}$ and $y \in W_{n-1}$ for some $n$. We can write (2.1) as follows:

$$
\delta_{\sigma(x) \sigma(y) \sigma(z)}=\frac{1}{2}\left(\delta_{\sigma(x) \sigma(y)}+\delta_{\sigma(y) \sigma(z)}\right) .
$$

The generalized Kroneker's function in (2.1) which corresponds to the quadratic stochastic operator is an identity transformation [14].

Model. In the Potts model, the spin variables $\sigma(x)$ take their values on a discrete set $\Phi=$ $\{1,2, \ldots, q\}, q>2$ which are associated with each vertex of the tree $J^{k}$ (see [15] for details).

The Potts model with three competing interactions and external field is defined by the following Hamiltonian

$$
H(\sigma)=-J \sum_{\langle x, y\rangle} \delta_{\sigma(x) \sigma(y)}-J_{1} \sum_{\rangle x, y\langle} \delta_{\sigma(x) \sigma(y)}-J_{2} \sum_{\langle x, y, z\rangle} \delta_{\sigma(x) \sigma(y) \sigma(z)}-h \sum_{x \in V} \delta_{0 \sigma(x)},
$$

where the first sum ranges within all nearest neighbors, the second sum ranges within all the second neighbors, the third sum ranges within all triples of neighbors and the spin variables $\sigma(x)$ may take the values $1,2,3 . J, J_{1}, J_{2} \in \mathbb{R}$ are coupling constants and $h$ is external field.

In [15], the authors have already obtained the exact solutions of the Potts model with competing ternary and binary interactions and external field on Cayley tree described by means of the Hamiltonian (2.2), where $h=0$ and $J_{1}=0$. In this paper, we assume that each parameter $J, J_{1}, J_{2}, h$ is different from zero. 


\section{Recursive equations for Partition Functions}

Let $\Lambda$ be a finite subset of $\mathrm{V}$. We will denote the restriction of $\sigma$ to $\Lambda$ by $\sigma(\Lambda)$. Let $\bar{\sigma}(\mathrm{V} \backslash \Lambda)$ be a fixed boundary configuration. The total energy of $\sigma(\Lambda)$ under condition $\bar{\sigma}(\mathrm{V} \backslash \Lambda)$ is defined as

$$
\begin{aligned}
H_{\Lambda}(\sigma(\Lambda) \mid \bar{\sigma}(V \backslash \Lambda))= & -J \sum_{\langle x, y\rangle: x, y \in \Lambda} \delta_{\sigma(x) \sigma(y)}-J \sum_{\langle x, y\rangle: x \in \Lambda, y \notin \Lambda} \delta_{\sigma(x) \sigma(y)} \\
& -J_{1} \sum_{>x, y\langle: x, y \in \Lambda} \delta_{\sigma(x) \sigma(y)}-J_{1} \sum_{>x, y\langle: x \in \Lambda, y \notin \Lambda} \delta_{\sigma(x) \sigma(y)} \delta_{\langle x, y, z\rangle: x, z \notin \Lambda, y \in \Lambda} \delta_{\sigma(x) \sigma(y) \sigma(z)} \\
& -J_{2} \sum_{\langle x, y, z\rangle: x, y, z \in \Lambda} \delta_{\sigma(x) \sigma(y) \sigma(z)}-J_{2} \sum \\
& -h \sum_{x \in \Lambda} \delta_{0 \sigma(x)} .
\end{aligned}
$$

Then, partition function $Z_{\Lambda}(\bar{\sigma}(V \backslash \Lambda))$ in volume $\Lambda$ under boundary condition $\bar{\sigma}(\mathrm{V} \backslash \Lambda)$ is defined as

$$
Z_{\Lambda}(\bar{\sigma}(V \backslash \Lambda))=\sum_{\sigma(\Lambda) \in \Omega(\Lambda)} \exp \left[-\beta H_{\Lambda}(\sigma(\Lambda) \mid \bar{\sigma}(V \backslash \Lambda))\right],
$$

where $\Omega(\Lambda)$ is the set of all configuration in volume $\Lambda$, and $\beta=1 / k T$ is the inverse temperature.

Instead of the configurations $\sigma\left(\mathrm{V}_{n}\right)$ and the partition functions $Z_{V_{n}}$ in volume $\mathrm{V}_{n}$ we use notations $\sigma_{n}$ and $\mathrm{Z}^{(n)}$, respectively 10,15$]$.

It is well known that the Gibbs measure is a probability measure used in many problems of probability theory and of statistical mechanics [13]. The Gibbs measure of a configuration $\sigma_{\Lambda}$ is defined by

$$
\mu\left(\sigma_{\Lambda}\right)=\frac{\exp \left[-\beta H\left(\sigma_{\Lambda}\right)\right]}{Z_{\Lambda}(\sigma)},
$$

where $Z_{\Lambda}(\sigma)$ is the partition function.

As usual, one can introduce the notion of the Gibbs measure (phase) of the Potts model with a competing interaction on the Cayley tree (see [6, 7, 13] for details).

Let us decompose the partition function $\mathrm{Z}^{(n)}$ into the following summands

$$
Z^{(n)}=\sum_{i=1}^{q} Z_{i}^{(n)}
$$

where

$$
Z_{i}^{(n)}=\sum_{\sigma_{n} \in \Omega\left(V_{n}\right): \sigma\left(x^{0}\right)=i} \exp \left[-\beta H_{V_{n}}\left(\sigma_{n} \mid \bar{\sigma}\left(V \backslash V_{n}\right)\right)\right] .
$$

Now the partition function is iteratively calculated by moving from the boundary towards to the interior of the Cayley tree of order two. The partial partition function of a branch on $n$ generations, where the innermost site is in state $i$, is denoted by $Z_{i}^{(n)}$.

Let $S\left(x^{0}\right)=\left\{x^{1}, x^{2}\right\}, \sigma\left(x^{0}\right)=i, \sigma\left(x^{1}\right)=j$ and $\sigma\left(x^{2}\right)=m$. For a given $n$, let us define the partial partition functions of the next generation by

$$
Z_{i}^{(n)}=\sum_{j, m=1}^{3} \exp \left[\beta J\left(\delta_{i j}+\delta_{i m}\right)+\beta J_{1} \delta_{j m}+\beta J_{2} \delta_{j i m}+h \delta_{1 i}\right] Z_{j}^{(n-1)} Z_{m}^{(n-1)},
$$

where $i=1,2,3$. 
Let $\theta=\exp (\beta J), \theta_{1}=\exp \left(\beta J_{1}\right), \theta_{2}=\exp \left(\beta J_{2}\right)$ and $\theta_{3}=\exp (\beta h)$. From (3.1) and (3.2), we can calculate the partial partition functions as follows:

$$
\begin{aligned}
Z_{1}^{(n)} & =\theta_{3}\left\{\theta^{2} \theta_{1} \theta_{2}\left(Z_{1}^{(n-1)}\right)^{2}+2 \theta \sqrt{\theta_{2}}\left(Z_{1}^{(n-1)} Z_{2}^{(n-1)}+Z_{1}^{(n-1)} Z_{3}^{(n-1)}\right)\right. \\
& \left.+\theta_{1}\left[\left(Z_{2}^{(n-1)}\right)^{2}+\left(Z_{3}^{(n-1)}\right)^{2}\right]+2 Z_{2}^{(n-1)} Z_{3}^{(n-1)}\right\} \\
Z_{2}^{(n)} & =\theta_{1}\left(Z_{1}^{(n-1)}\right)^{2}+2 \theta \sqrt{\theta_{2}} Z_{2}^{(n-1)}\left(Z_{1}^{(n-1)}+Z_{3}^{(n-1)}\right) \\
& +2 Z_{1}^{(n-1)} Z_{3}^{(n-1)}+\theta^{2} \theta_{1} \theta_{2}\left(Z_{2}^{(n-1)}\right)^{2}+\theta_{1}\left(Z_{3}^{(n-1)}\right)^{2} \\
Z_{3}^{(n)} & =\theta_{1}\left(Z_{1}^{(n-1)}\right)^{2}+2 \theta \sqrt{\theta_{2}} Z_{3}^{(n-1)}\left(Z_{1}^{(n-1)}+Z_{2}^{(n-1)}\right)^{2} \\
& +2 Z_{1}^{(n-1)} Z_{2}^{(n-1)}+\theta^{2} \theta_{1} \theta_{2}\left(Z_{3}^{(n-1)}\right)^{2}+\theta_{1}\left(Z_{2}^{(n-1)}\right)^{2} .
\end{aligned}
$$

After replacement $u_{n}=Z_{2}^{(n)} / Z_{1}^{(n)}$ and $v_{n}=Z_{3}^{(n)} / Z_{1}^{(n)}$ we have the following system of recurrent equations

$$
\begin{aligned}
\theta_{3} u_{n} & =\frac{\theta_{1}+2 \theta \sqrt{\theta_{2}} u_{n-1}\left(1+v_{n-1}\right)+2 v_{n-1}+\theta^{2} \theta_{1} \theta_{2} u_{n-1}^{2}+\theta_{1} v_{n-1}^{2}}{\theta^{2} \theta_{1} \theta_{2}+2 \theta \sqrt{\theta}_{2}\left(u_{n-1}+v_{n-1}\right)+\theta_{1}\left(u_{n-1}^{2}+v_{n-1}^{2}\right)+2 u_{n-1} v_{n-1}} \\
\theta_{3} v_{n} & =\frac{\theta_{1}+2 u_{n-1}+2 \theta \sqrt{\theta_{2}} v_{n-1}\left(1+u_{n-1}\right)+\theta_{1} u_{n-1}^{2}+\theta^{2} \theta_{1} \theta_{2} v_{n-1}^{2}}{\theta^{2} \theta_{1} \theta_{2}+2 \theta \sqrt{\theta}{ }_{2}\left(u_{n-1}+v_{n-1}\right)+\theta_{1}\left(u_{n-1}^{2}+v_{n-1}^{2}\right)+2 u_{n-1} v_{n-1}} .
\end{aligned}
$$

We define the transformation

$$
\mathbf{F}=\left(F_{1}, F_{2}\right): \mathbb{R}^{2} \rightarrow \mathbb{R}^{2}
$$

with $u_{n}=F_{1}\left(u_{n-1}, v_{n-1}\right)$ and $v_{n}=F_{2}\left(u_{n-1}, v_{n-1}\right)$. The fixed points of the function (3.5) $\mathbf{w}=$ $\mathbf{F}(\mathbf{w})$, where $\mathbf{w}=\left(u_{n}, v_{n}\right)$, describe translation-invariant Gibbs measures (phases) of the Potts model defined by the Hamiltonian (2.2).

The recursive equations (3.4) can be written as $w_{n}=\mathbf{F}\left(w_{n-1}\right), n>0$. In the theory of dynamical systems, $w_{n}$ is called trajectory of the initial point $w_{1}$ under the action of the mapping F. So, the asymptotic behavior of $Z^{(n)}$ for $n \rightarrow \infty$ can be determined by value of $\lim _{n} w_{n}$.

If $u=\lim _{n} u_{n}$ and $v=\lim _{n} v_{n}$ then we have the following equations;

$$
\begin{aligned}
& \theta_{3} u=\frac{\theta_{1}+2 \theta \sqrt{\theta_{2}} u(1+v)+2 v+\theta^{2} \theta_{1} \theta_{2} u^{2}+\theta_{1} v^{2}}{\theta^{2} \theta_{1} \theta_{2}+2 \theta \sqrt{\theta}_{2}(u+v)+\theta_{1}\left(u^{2}+v^{2}\right)+2 u v} \\
& \theta_{3} v=\frac{\theta_{1}+2 u+2 \theta \sqrt{\theta_{2}} v(1+u)+\theta_{1} u^{2}+\theta^{2} \theta_{1} \theta_{2} v^{2}}{\theta^{2} \theta_{1} \theta_{2}+2 \theta \sqrt{\theta}_{2}(u+v)+\theta_{1}\left(u^{2}+v^{2}\right)+2 u v} .
\end{aligned}
$$

To describe phases (Gibbs measures) of a given Hamiltonian on a Cayley tree, one has a correspondence between Gibbs measures and a collection of vectors $\left\{h_{x}, x \in V\right\}$, which satisfy a non-linear equation. The recursive equations (3.6) considered in this paper describe a vector function $\left\{\left(u_{n}, v_{n}\right), n \in \mathbb{N}\right\}$ which is a particular case of the above mentioned function $h_{x}$ obtained as $h_{x}=u_{n}$ if $x \in W_{n}$ i.e., depends only on the number of the generation set to which belongs $x$ but not on $x$ itself (see for example, [12, 15]).

The solutions of the system (3.6) describe the translation-invariant Gibbs measures [6]. The number of the solutions of the equations (3.6) depends on the parameters $\beta=1 / k T, \theta, \theta_{1}, \theta_{2}$, $\theta_{3}$ and $h$. The phase transition usually occurs for low temperature. It is possible to find an exact value of $T^{*}$ such that a phase transition occurs for all $T<T^{*}$ where $T^{*}$ is called a critical value of temperature.

An attractive fixed point of a function $f$ is a fixed point $u_{0}$ of $f$ such that for any value of $u$ in a domain that is close enough to $u_{0}$, the iterated function sequence $u, f(u), f(f(u)), f(f(f(u))), \ldots$ converges to $u_{0}$. An attractive fixed point is said to be a stable fixed point if it is also Lyapunov stable (see [18] for details). 


\section{Translation-invariant Gibbs measures}

As stated in [6], for a given potential $V$ we have exactly one Gibbs state with potential $V$ if the graph is finite. For some potentials there may be more than one Gibbs state if the graph is not finite. When we have more than one Gibbs state, then we say that there exists a phase transition for a given potential $V$. In other words, this occurrence of non-uniqueness of a Gibbs measure can be interpreted as a phase transition.

In this section we determine whether any phase transition occurs or not by solving the system (3.6). If there is more than one positive solution for the equations (3.6), then there is one Gibbs measure [16] corresponding to each of these solutions. If there is more than one Gibbs measure, then it is said that a phase transition occurs for this model (2.2) [12, 15].

\subsection{First case}

Assume that $u=v$, then some solutions of the system (3.6) can be found from equation

$$
\theta_{3} u=\frac{\left(\tilde{\theta}^{2} \theta_{1}+2 \tilde{\theta}+\theta_{1}\right) u^{2}+2(\tilde{\theta}+1) u+\theta_{1}}{2\left(\theta_{1}+1\right) u^{2}+4 \tilde{\theta} u+\tilde{\theta}^{2} \theta_{1}}:=f(u),
$$

where $\tilde{\theta}=\theta \sqrt{\theta_{2}}$.

In this case, in order to obtain the solutions of the system (3.6), we should analyze the equation in (4.1).

1. When we take the first derivative of $f(u)$, we see that if $\tilde{\theta}>1$, that is $\theta_{2}>1 / \theta^{2}$, then $f(u)$ is increasing and if $\tilde{\theta}<1, f(u)$ is decreasing for $u>0$;

2. When we take the second derivative of $f(u)$, we release that if $\theta_{1}>\theta_{1}^{*}$, where

$$
\theta_{1}^{*}=\frac{\tilde{\theta}^{2}+\tilde{\theta}+1+\sqrt{9 \tilde{\theta}^{4}+26 \tilde{\theta}^{3}+35 \tilde{\theta}^{2}+50 \tilde{\theta}+33}}{\left(\tilde{\theta}^{2}+2\right)(\tilde{\theta}+1)}
$$

then there is an inflection point $u^{*}>0$ such that $f^{\prime \prime}(u)>0$ for $0<u<u^{*}$ and $f^{\prime \prime}(u)<0$ for $u>u^{*}$ (see [6, Proposition 10.7] for details).

Theorem 1 [19, Theorem 5.1] Suppose that $f^{\prime \prime}$ exists on an interval $I$.

(i) If $f^{\prime \prime}(u)>0$ on $I$, then the graph of $f$ is concave upward on $I$.

(ii) If $f^{\prime \prime}(u)<0$ on $I$, then the graph of $f$ is concave downward on $I$.

Thus we can generalize the Lemma proved in [2] to the model (2.2) as follows.

Lemma 1 Assume that the quartic polynomial $g$ in (4.2) has two positive real roots. Let $\tilde{\theta}>1$, $\theta_{2}>1 / \theta^{2}$, that is the equation $f$ in (4.1) has a positive inflection point. Then, there exist $\eta_{1}\left(\theta, \theta_{1}, \theta_{2}\right)$ and $\eta_{2}\left(\theta, \theta_{1}, \theta_{2}\right)$ with $0<\eta_{1}\left(\theta, \theta_{1}, \theta_{2}\right)<\eta_{2}\left(\theta, \theta_{1}, \theta_{2}\right)$ such that the equation (4.1) has three positive roots $u_{*}^{(1)}<u_{*}^{(2)}<u_{*}^{(3)}$ if $\eta_{1}\left(\theta, \theta_{1}, \theta_{2}\right)<\theta_{3}<\eta_{2}\left(\theta, \theta_{1}, \theta_{2}\right)$; has two solutions if either $\theta_{3}=\eta_{1}\left(\theta, \theta_{1}, \theta_{2}\right)$ or $\theta_{3}=\eta_{2}\left(\theta, \theta_{1}, \theta_{2}\right)$ and has a single solution in other cases. In fact

$$
\eta_{i}\left(\theta, \theta_{1}, \theta_{2}\right)=\frac{1}{u_{i}^{*}} f\left(u_{i}^{*}\right)
$$

where $u_{1}^{*}, u_{2}^{*}$ are the solutions of equation $u f^{\prime}(u)=f(u)$.

Proof follows from properties 1) and 2) of function $f$. As mentioned in [6, Proposition 10.7], for the equation (4.1) there is more than one solution if and only if there is more than one solution to $u f^{\prime}(u)=f(u)$, which is equivalent to the following equation

$$
g(u)=A u^{4}+B u^{3}+C u^{2}+D u+E=0,
$$


where $A=2\left(\theta_{1}+1\right)\left(\tilde{\theta}^{2} \theta_{1}+2 \tilde{\theta}+\theta_{1}\right)>0, B=8(\tilde{\theta}+1)\left(\theta_{1}+1\right)>0, D=8 \tilde{\theta} \theta_{1}>0, E=\tilde{\theta}^{2} \theta_{1}>0$ and

$$
C=-\left(\tilde{\theta}^{4}+\tilde{\theta}^{2}-6\right) \theta_{1}^{2}-\left(2 \tilde{\theta}^{3}-6\right) \theta_{1}+8 \tilde{\theta}^{2} .
$$

Let us determine where the graph of $g$ given in (4.2) is concave upward and concave downward. Here, we have

$$
g^{\prime}(u)=4 A u^{3}+3 B u^{2}+2 C u+D .
$$

We now have $g^{\prime \prime}(u)=12 A u^{2}+6 B u+2 C$. Assume that

$$
3 B^{2}-8 A C>0 .
$$

From Theorem $1 g$ is concave downward on interval

$$
I=\left(\frac{-3 B-\sqrt{9 B^{2}-24 A C}}{12 A}, \frac{-3 B+\sqrt{9 B^{2}-24 A C}}{12 A}\right)
$$

and $g$ is concave upward on interval $\mathbb{R} \backslash I$. Notice that at the point

$$
\left(\frac{-3 B+\sqrt{9 B^{2}-24 A C}}{12 A}, g\left(\frac{-3 B+\sqrt{9 B^{2}-24 A C}}{12 A}\right)\right),
$$

the graph chances from concave downward to concave upward. Thus,

$$
\left(\frac{-3 B+\sqrt{9 B^{2}-24 A C}}{12 A}, g\left(\frac{-3 B+\sqrt{9 B^{2}-24 A C}}{12 A}\right)\right)
$$

is one of the inflection points of $g$.

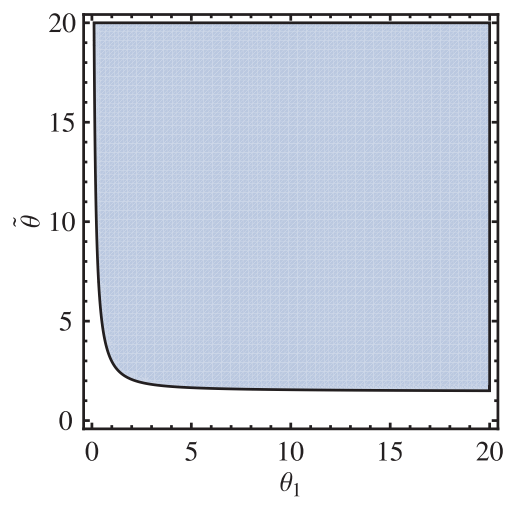

Figure 1. The region satisfying the inequality (4.4).
It is clear that $g(0)=E>0$ and $\lim _{u \rightarrow \infty} g(u)=\infty$. Since $g$ is a continuous function, we can conclude that if $g(u)<0$ on $J \subset \mathbb{R}^{+}$, then $g$ has two positive roots under condition 4.3).

Due to Descartes' Rule of Signs [17], the maximum number of positive real roots of the quartic polynomial $g$ in (4.2) can be found by counting the number of sign changes in the equation (4.2). Thus, the equation (4.2) has no positive roots if $C>0$, and the polynomial (4.2) has at most two positive roots $u_{1}^{*}$, $u_{2}^{*}$ if $C<0$. In this case, if $\tilde{\theta}>\sqrt{2}$ and $\left(2 \tilde{\theta}^{3}-6\right)^{2}+32 \tilde{\theta}^{2}\left(\tilde{\theta}^{4}+\tilde{\theta}^{2}-6\right)<0$ then the coefficient $C$ is negative.

Due to

$$
0=A u^{4}+B u^{3}+C u^{2}+D u+E \geqslant A u^{4}+C u^{2}+E \geqslant 2 \sqrt{A u^{4} E}+C u^{2}=(2 \sqrt{A E}+C) u^{2}
$$

from this we get $2 \sqrt{A E}+C \leqslant 0$ i.e. $C \leqslant-2 \sqrt{A E}$, this simple calculation shows that a necessary condition to have positive roots of the equation (4.2) is $C<-2 \sqrt{A E}$. Thus, we have the following inequality:

$$
8 \tilde{\theta}+8 \tilde{\theta}^{2}+6 \theta_{1}-2 \tilde{\theta}^{3} \theta_{1}+6 \theta_{1}^{2}-\tilde{\theta}^{2} \theta_{1}^{2}-\tilde{\theta}^{4} \theta_{1}^{2}+2 \sqrt{\tilde{\theta}^{2} \theta_{1}\left(1+\theta_{1}\right)\left(2 \tilde{\theta}+\theta_{1}+\tilde{\theta}^{2} \theta_{1}\right)}<0 .
$$

Let us define the set $H=\left\{\left(\theta_{1}, \tilde{\theta}\right): C<-2 \sqrt{A E}\right\}$ (see figure 1).

Then, we have the following Corollary. 
Corollary 1 If $\theta_{2}>1 / \theta^{2}, 3 B^{2}-8 A C>0,\left(\theta_{1}, \tilde{\theta}\right) \in H$ and $\eta_{1}\left(\theta, \theta_{1}, \theta_{2}\right)<\theta_{3}<\eta_{2}\left(\theta, \theta_{1}, \theta_{2}\right)$ then the function $f$ given in (4.1) has three fixed points. Thus, for the model (2.2) there are three translation-invariant Gibbs measures $\mu_{1}, \mu_{2}, \mu_{3}$ indicating a phase transition.

It is easy to show that these three measures correspond to boundary conditions $\bar{\sigma}\left(V \backslash V_{n}\right) \equiv i$, $i=1,2,3$.

In figure 1 we show the region with three positive roots. For parameters $\tilde{\theta}, \theta_{1}$ in phase transition region the equation (4.1) has three positive roots, where two of them are stable and the third one is unstable.

\subsubsection{Illustrative example}

Using the Mathematica software, we have manipulated the function $f / \theta_{3}$ given in (4.1) for some parameters $\theta, \theta_{1}, \theta_{2}$ and $\theta_{3}$. In figure 2, for $\theta=0.2, \theta_{1}=64, \theta_{2}=2.2$ and $\theta_{3}=1.6$ we have only one fixed point. Thus, in this case the model (2.2) has no phase transition.

If we take as $\theta=11.6, \theta_{1}=28.1, \theta_{2}=10.1, \theta_{3}=12.2$, then we have two fixed points. As shown figure 2 (c), if we take as $\theta=10.3, \theta_{1}=6.49, \theta_{2}=6.9$ and $\theta_{3}=6.4$, then we have three fixed points, where two of them are stable and the third one is unstable.
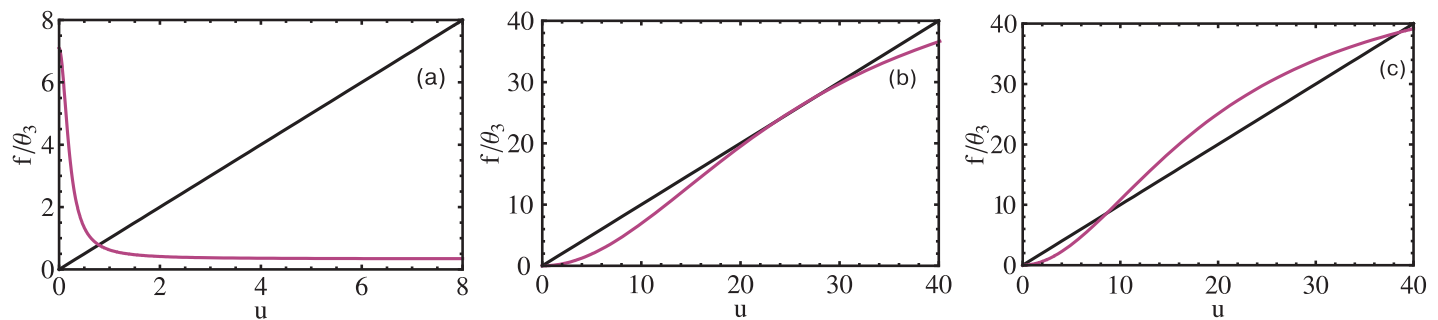

Figure 2. Graphs of the function $f / \theta_{3}$ given in (4.1) (a) for $\theta=0.2, \theta_{1}=64, \theta_{2}=2.2$ and $\theta_{3}=1.6$; (b) $\theta=11.6, \theta_{1}=28.1, \theta_{2}=10.1, \theta_{3}=12.2$; (c) $\theta=10.3, \theta_{1}=6.49, \theta_{2}=6.9$ and $\theta_{3}=6.4$, respectively.

Thus, in order to study the phase transition for the model (2.2) we have clarified the role of $\theta$, $\theta_{1}, \theta_{2}$ and $\theta_{3}$.

\subsection{Second case}

Now, let us find the other solutions of the system (3.6). Assume that $u \neq v$. Subtracting the second equation of (3.6) from the first we have

$$
\theta_{3}(u-v)=\frac{2(\tilde{\theta}-1)(u-v)+\theta_{1}\left(\tilde{\theta}^{2}-1\right)\left(u^{2}-v^{2}\right)}{\tilde{\theta}^{2} \theta_{1}+2 \tilde{\theta}(u+v)+2 u v+\theta_{1}\left(u^{2}+v^{2}\right)} .
$$

After canceling to $(u-v)$ two sides of the equation (4.5) we can obtain the following equation;

$$
t=\frac{\theta_{1} \theta_{2} s^{2}+\left[2 \theta_{2} \tilde{\theta}-\theta_{1}\left(\tilde{\theta}^{2}-1\right)\right] s+\tilde{\theta}^{2} \theta_{1} \theta_{2}-2(\tilde{\theta}-1)}{2 \theta_{3}\left(\theta_{1}-1\right)},
$$

where $u+v=s$ and $u v=t$.

After dividing the first equation of (3.6) to the second and simplifying we have

$$
t=\frac{\theta_{1} s^{2}+2 s+\theta_{1}}{\tilde{\theta}^{2} \theta_{1}+\theta_{1}-2 \tilde{\theta}} .
$$


From (4.6) and (4.7) we obtain the following equality;

$$
\begin{aligned}
\frac{\theta_{1}(\tilde{\theta}+1) s+2}{\theta_{3}}= & \frac{\theta_{1}\left[\theta_{1}(\tilde{\theta}+1)-2\right] s^{2}+2\left[\theta_{1}\left(\tilde{\theta}^{2}+\tilde{\theta}-2\right)-2(\tilde{\theta}+1)\right] s}{\theta_{1}\left(\tilde{\theta}^{2}+1\right)-2 \tilde{\theta}} \\
& +\frac{\theta_{1}\left[\theta_{1}\left(\tilde{\theta}^{3}+\tilde{\theta}^{2}+2 \tilde{\theta}+2\right)-2\left(\tilde{\theta}^{2}+\tilde{\theta}+1\right)\right]}{\theta_{1}\left(\tilde{\theta}^{2}+1\right)-2 \tilde{\theta}} .
\end{aligned}
$$

Let us consider a system of equations

$$
\begin{aligned}
u+v & =s \\
u v & =t .
\end{aligned}
$$

From (4.9), we can write $v=s-u$ and $u(s-u)=t$, thus we have

$$
u^{2}-s u+t=0 \text {. }
$$

To have two different real roots of the second-order equation (4.10), inequality $s^{2}-4 t>0$ should be valid. Then the equations (4.9) have solutions, if $t<s^{2} / 4$.

Thus, from (4.7) and from the inequality $t<s^{2} / 4$ we can get the following equation

$$
\left[\theta_{1}\left(\tilde{\theta}^{2}-3\right)-2 \tilde{\theta}\right] s^{2}-8 s-4 \theta_{1}>0 .
$$

Then, there is

$$
s^{*}=\frac{4+\sqrt{16+4 \theta_{1}\left[\theta_{1}\left(\tilde{\theta}^{2}-3\right)-2 \tilde{\theta}\right]}}{\theta_{1} \tilde{\theta}^{2}-3 \theta_{1}-2 \tilde{\theta}}>0,
$$

such that for all $s>s^{*}$ the inequality $t<s^{2} / 4$ is valid.

One can show that the inequality (4.11) is satisfied, if

$$
\left(\tilde{\theta}^{2}-3\right) \theta_{1}^{2}-2 \tilde{\theta} \theta_{1}+4<0
$$

and $\theta_{1}\left(\tilde{\theta}^{2}-3\right)-2 \tilde{\theta}>0$. Thus, for $\tilde{\theta}>\sqrt{3}$ and

$$
0<\theta_{1}<\frac{\tilde{\theta}+\sqrt{12-3 \tilde{\theta}^{2}}}{\tilde{\theta}^{2}-3}
$$

the inequality (4.12) is valid.

In this case, the system of the equations (4.9) has solutions.

Assume that all coefficients of the equation (4.8) are positive. With the help of the elementary analysis we obtain the following three cases:

1. If

$$
\theta_{3}<\frac{2\left[\theta_{1}\left(\tilde{\theta}^{2}+1\right)-2 \tilde{\theta}\right]}{\theta_{1}\left[\theta_{1}\left(\tilde{\theta}^{3}+\tilde{\theta}^{2}+2 \tilde{\theta}+2\right)-2\left(\tilde{\theta}^{2}+\tilde{\theta}+1\right)\right]},
$$

then the equation (4.8) has only one positive root.

2. If

$$
\theta_{3}>\frac{2\left[\theta_{1}\left(\tilde{\theta}^{2}+1\right)-2 \tilde{\theta}\right]}{\theta_{1}\left[\theta_{1}\left(\tilde{\theta}^{3}+\tilde{\theta}^{2}+2 \tilde{\theta}+2\right)-2\left(\tilde{\theta}^{2}+\tilde{\theta}+1\right)\right]}
$$

there is $k_{0}$ such that the line $y-2 / \theta_{3}=k_{0} s$ is a tangent for the parabola on the right-hand side of (4.8) and then for $\theta_{3}<\theta_{1}(\tilde{\theta}+1) / k_{0}$ the equation (4.8) has two positive roots and only one of them is greater than $s^{*}$. 
3. The equation (4.8) has two positive roots, when

$$
\theta>\sqrt{\frac{3}{\theta_{2}}}, \quad 0<\theta_{1}<\frac{\tilde{\theta}+\sqrt{12-3 \tilde{\theta}^{2}}}{\tilde{\theta}^{2}-3}, \quad \theta_{1}>\frac{2 \tilde{\theta}}{\tilde{\theta}^{2}-3}
$$

and

$$
\frac{2\left[\theta_{1}\left(\tilde{\theta}^{2}+1\right)-2 \tilde{\theta}\right]}{\theta_{1}\left[\theta_{1}\left(\tilde{\theta}^{3}+\tilde{\theta}^{2}+2 \tilde{\theta}+2\right)-2\left(\tilde{\theta}^{2}+\tilde{\theta}+1\right)\right]}<\theta_{3}<\frac{\theta_{1}(\tilde{\theta}+1)}{k_{0}}
$$

where $k_{0}$ is defined as above.

\subsubsection{Illustrative example}

Let us give an illustrative example. With the help of the Mathematica software, we have manipulated the line on the left-hand side and the parabola on the right-hand side of the equation (4.8) for some parameters $\theta, \theta_{1}, \theta_{2}$ and $\theta_{3}$. We have found the points of intersection of the parabola and the line given in (4.8).

In figure 3 (a), the inequality (4.14) is satisfied, thus we have only one positive root. In figure 3 (b), the line $y-2 / \theta_{3}=\theta_{1}(\tilde{\theta}+1) s / \theta_{3}$ is a tangent for the parabola (4.8). In figure 3 (c), the inequality (4.16) is satisfied, we have two positive solutions. Thus, in order to obtain the solutions of the system (4.9), we have clarified the role of the parameters $\theta, \theta_{1}, \theta_{2}$ and $\theta_{3}$.
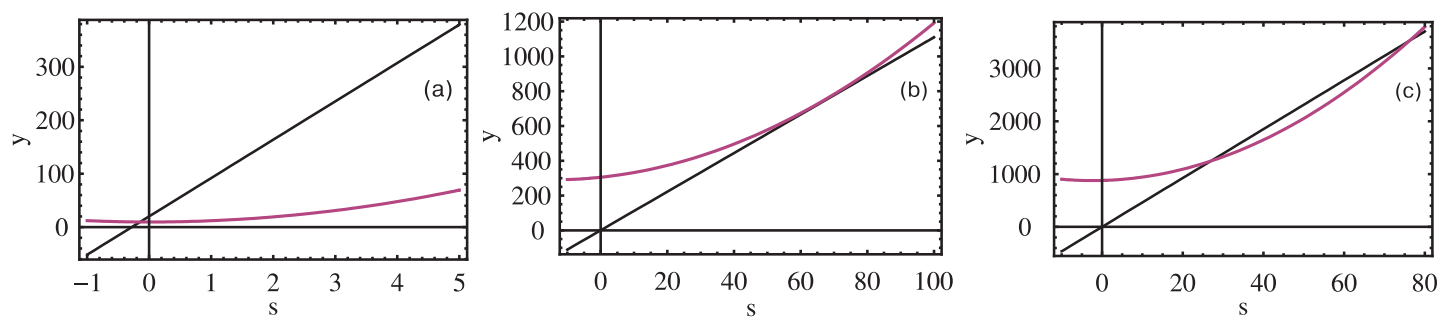

Figure 3. Graphs of the parabola and the line given in (4.8) a) for $\theta=1.1, \theta_{1}=2.9, \theta_{2}=1.8$ and $\theta_{3}=1.1$; b) $\theta=27.5, \theta_{1}=4.5, \theta_{2}=5.9$ and $\theta_{3}=27.5$; c) $\theta=6.42, \theta_{1}=19, \theta_{2}=50$ and $\theta_{3}=19$, respectively.

Recalling the results for the equations (3.6), we get the following result: if $\left(\theta, \theta_{1}, \theta_{2}\right) \in M$ and $K<\theta_{3}<L$, where

$$
\begin{aligned}
M & =\left\{\left(\theta, \theta_{1}, \theta_{2}\right): \theta>\sqrt{\frac{3}{\theta_{2}}}, \theta_{1}>\frac{2 \tilde{\theta}}{\left(\tilde{\theta}^{2}-3\right)}, 0<\theta_{1}<\frac{\tilde{\theta}+\sqrt{12-3 \tilde{\theta}^{2}}}{\tilde{\theta}^{2}-3}\right\} \cap H \\
K & =\max \left(\eta_{1}, \frac{2\left[\theta_{1}\left(\tilde{\theta}^{2}+1\right)-2 \tilde{\theta}\right]}{\theta_{1}\left[\theta_{1}\left(\tilde{\theta}^{3}+\tilde{\theta}^{2}+2 \tilde{\theta}+2\right)-2\left(\tilde{\theta}^{2}+\tilde{\theta}+1\right)\right]}\right) \\
L & =\min \left(\eta_{2}, \frac{\theta_{1}(\tilde{\theta}+1)}{k_{0}}\right)
\end{aligned}
$$

then the system (3.6) has five positive solutions, three of which are obtained from the first case and the other two solutions arise in the second case. Similar to [2] and [20], a more detailed analysis shows that only three solutions are stable.

Combining the first and the second cases, we have proved the following theorem.

Theorem 2 Assume that the conditions (4.17) are satisfied, then for the model (2.2) there are five translation-invariant Gibbs measures indicating a phase transition. 
Remark 1 In a similar way we can study the solutions of a new system of nonlinear equations by using Kroneker's symbol $\delta$ that has the form

$$
\delta_{\sigma(x) \sigma(y) \sigma(z)}= \begin{cases}1, & \text { if } \sigma(x)=\sigma(y)=\sigma(z) \\ 0, & \text { otherwise. }\end{cases}
$$

\section{Conclusion}

In this paper we have exactly solved the Potts model on a Cayley tree, the Hamiltonian of which contains three competing interactions, the nearest neighbors, the second neighbors, triples of neighbors and the external field. Namely, we have calculated the critical curve such that there is a phase transition above it, and a single Gibbs state is found elsewhere. We have clarified the role of the coupling constants $J, J_{1}, J_{2}$ and external field $h$ to study the existence problem of a phase transition. We have considered transformation $\mathbf{F}=\left(F_{1} ; F_{2}\right): \mathbb{R}^{2} \rightarrow \mathbb{R}^{2}$ and proved that its fixed points describe the translation-invariant Gibbs measures of our model. We have seen that for some values $J, J_{1}, J_{2}$ and $h$, the phase transition occurs.

In [12], an Ising model with four competing interactions (external field, nearest neighbor, second neighbors and triples of neighbors) on the Cayley tree of order two has been solved. The authors [12] have also constructed numerous non-periodic extreme Gibbs measures. The investigation of the periodic Gibbs measures for the Potts model (2.2) is planned to be the subject of forthcoming publications.

\section{Acknowledgement}

The authors would like to thank Prof. Dr. N.N. Ganikhodjaev and Prof. Dr. Utkir Rozikov for valuable support and suggestions. We are also grateful to anonymous referees whose comments greatly improved the paper. 


\title{
References
}

1. Wu F.Y., Rev. Mod. Phys., 1982, 54, 235-268; doi 10.1103/RevModPhys.54.235

2. Ganikhodjaev N.N., Akin H., Temir S., Turk. J. Math., 2007, 31, 229-238.

3. Ganikhodjaev N.N., Temir S., Akin H., J. Stat. Phys., 2009, 137, 701-715; doi $10.1007 / \mathrm{s} 10955-009-9869-\mathrm{z}$.

4. Akin H., Temir S., Int. J. Appl. Math. Mech., 2008, 4, No 6, 71-77.

5. Baxter R.J., Exactly Solved Models in Statistical Mechanics. Academic Press, London, 1982.

6. Preston K., Gibbs States on Countable Sets. Cambridge Univ. Press, London, 1974.

7. Sinai Ya.G., Theory of Phase Transitions: Rigorous Results. Pergamon, Oxford, 1982.

8. da Silva C.R., and Countiho S., Phys. Rev. B, 1986, 34, 7975-7985; doi 10.1103/PhysRevB.34.7975

9. Spitzer F., Ann. Probability, 1975, 3, 387-398; doi 10.1214/aop/1176996347

10. Ganikhodjaev N.N., Pah C.H., Wahiddin M.R., J. Math. Phys., 2004, 45, 3645-3658; doi:10.1063/1.1781747.

11. Kindermann R., Snell J.L., Markov Random Fields and Their Applications, Contemporary Mathematics, 1, Providence, R. I., Amer. Math. Soc., 1980.

12. Ganikhodjaev N.N., Rozikov U.A., Math. Phys. Anal. Geom., 2009, 12 (2), 141-156; doi: $10.1007 / \mathrm{s} 11040-009-9056-0$.

13. Georgii H.O., Gibbs Measures and Phase Transitions, De Gruyter studies in Mathematics, 9. Berlin, New York, 1988.

14. Mariz A.M., Tsallis C., Albuquerque E.L., J. Stat. Phys., 1985, 40, 577-592; doi: $10.1007 / \mathrm{BF} 01017186$

15. Ganikhodjaev N.N., Temir S., Akin H., CUBO Math. J., 2005, 7, 37-48.

16. Mukhamedov F., Rozikov, U., J. Stat. Phys., 2005, 119, 427-446; doi 10.1007/s10955-004-2056-3.

17. Hall H.S., Knight S.R., Higher Algebra: A Sequel to Elementary Algebra for Schools. Macmillan, London, 1950.

18. Devaney R.L., An Introduction to Chaotic Dynamical Systems. Benjamin/Cummings, Menlo Park, CA, 1986.

19. Smith R.T., Minton R.B., Calculus. 2 ed., McGraw-Hill Science/Engineering/Math, 2002.

20. Ganikhodjaev N.N., Teor. Mat. Fiz., 1990, 85, 163-175 (in Russion); [Engl. transl.: Theor. Math. Phys., 1990, 85, 1125-1134; doi 10.1007/BF01086840.

\section{До фазових переходів моделі Поттса з трьома конкуруючими взаємодіями на дереві Келі}

\author{
Г. Акін1, С. Темір 2 \\ 1 Університет Зірве, 27260 Газіантеп, Туреччина \\ 2 Університет Гарран, 63200 Санліурфа, Туреччина
}

У цій статті ми вивчаємо проблему фазового переходу для моделі Поттса з трьома конкуруючими взаємодіями, найближчих сусідів, наступних близьких сусідів і наступних за наступними близькими сусідами, з ненульовим зовнішнім полем на дереві Келі другого порядку. Ми доводимо, що для деяких значень параметрів моделі $є$ фазовий перехід. Ми приводимо проблему опису граничних мір Гіббса до проблеми розв'язку системи нелінійних функціональних рівнянь. Ми розширюємо результати, отримані Ганіходяєвим і Розіковим [Math. Phys. Anal. Geom., 2009, 12, No 2, 141-156] для фазового переходу в моделі Ізінга, на випадок моделі Поттса.

Ключові слова: фазовий перехід, модель Поттса, конкуруючі взаємодії, міра Гіббса 
